\title{
Response to "On pre- or postnatal diagnostic X-rays as a risk factor for childhood cancer review" by $R$. Wakeford
}

\author{
R. Schulze-Rath $\cdot$ G. P. Hammer • M. Blettner
}

Received: 15 January 2009 / Accepted: 7 February 2009 / Published online: 26 February 2009

(C) Springer-Verlag 2009

We thank Dr. Wakeford for his comments (Wakeford 2009) on our article "Are pre- or postnatal diagnostic X-rays a risk factor for childhood cancer? A systematic review." (Schulze-Rath et al. 2008).

His comment on our systematic review is very interesting and we generally agree with his findings. Nevertheless, it is noted that the scope of our review differed from that of Wakeford's review (Wakeford 2008). We did not focus only on prenatal but also on postnatal exposure with diagnostic X-rays. Furthermore, Wakeford's publication only includes prenatal maternal abdominal X-ray exposures, while ours includes any prenatal maternal $\mathrm{X}$-ray exposure, because the data in the included studies did not allow any sufficient and more detailed analyses. Thus, the odds ratio (OR) of 1.16 does not compare directly to the one of 0.99 that we found. The difference between the two ORs suggests that maternal abdominal X-rays may have a higher cancer risk for the child.

The differences in the literature included in the two publications (Schulze-Rath et al. 2008; Wakeford 2008) may be explained as follows: our inclusion criteria for the literature search were narrower. Studies were included if the publication was written in English and an effect estimate as OR or relative risk (RR) was reported. Additionally, the studies had to be published between 1990 and 2005. We did not include studies with overlapping cohorts. Two publications were not included as they are based on the same data as more recent publications. One article was not detailed enough to abstract a $2 \times 2$ table, and one was in Spanish. Three articles were not found by our search. In our original article, we already noted that literature databases often lack appropriate keywords for epidemiological studies, which makes it difficult to complete a systematic review. As a result, some of the studies included in our review we found in the reference lists of other publications only.

We totally agree that the current data are unsatisfactory and that they do not allow any further detailed analyses. In no way should our analysis be interpreted as suggesting no risk for pre-or postnatal diagnostic X-ray procedures.

\section{References}

Schulze-Rath R, Hammer GP, Blettner M (2008) Are pre- or postnatal diagnostic X-rays a risk factor for childhood cancer? A systematic review. Radiat Environ Biophys 47:301-312

Wakeford R (2008) Childhood leukaemia following medical diagnostic exposure to ionising radiation in utero or after birth. Radiat Prot Dosim 132(2): 166-174. doi:10.1093/rpd/ncn272

Wakeford R (2009) On pre- or postnatal diagnostic X-rays as a risk factor for chilchood cancer. Radiat Environ Biophys. doi:10. 1007/s00411-009-0214-3

R. Schulze-Rath $(\bowtie) \cdot$ G. P. Hammer · M. Blettner

Institute for Medical Biostatistics,

Epidemiology and Informatics,

Johannes-Gutenberg-University Mainz,

55109 Mainz, Germany

e-mail: schulzerath@imbei.uni-mainz.de 